\title{
On the Design of Experiments for the Study of Relativistic Nonlinear Optics in the Limit of Single-Cycle Pulse Duration and Single-Wavelength Spot Size ${ }^{1}$
}

\author{
G. Mourou1, Z. Chang1, A. Maksimchuk' ${ }^{1}$, J. Nees', S. V. Bulanov ${ }^{2}$, V. Yu. Bychenkov ${ }^{3}$, \\ T. Zh. Esirkepov ${ }^{4}$, N. M. Naumova ${ }^{2}$, F. Pegoraro ${ }^{5}$, and H. Ruhl ${ }^{6}$ \\ ${ }^{1}$ Center for Ultrafast Optical Science, University of Michigan, Ann Arbor, MI, USA \\ ${ }^{2}$ Institute of General Physics, Russian Academy of Sciences, ul. Vavilova 38, Moscow, 119991 Russia \\ ${ }^{3}$ Lebedev Institute of Physics, Russian Academy of Sciences, Leninskil pr. 53, Moscow, 119991 Russia \\ ${ }^{4}$ Moscow Institute for Physics and Technology, Institutskiŭ per. 9, Dolgoprudnyı̆, Moscow oblast, 141700 Russia \\ ${ }^{5}$ University of Pisa and National Institute for Physics of Matter, Pisa, Italy \\ ${ }^{6}$ Max Born Institute, Berlin, Germany \\ Received June 18, 2001
}

\begin{abstract}
We propose a set of experiments with the aim of studying for the first time relativistic nonlinear optics in the fundamental limits of single-cycle pulse duration and single-wavelength spot size. The laser system that makes this work possible is now operating at the Center for Ultrafast Optical Science at the University of Michigan. Its high repetition rate $(1 \mathrm{kHz})$ will make it possible to perform a detailed investigation of relativistic effects in this novel regime. This study has the potential to make the field of relativistic optics accessible to a wider community and to open the door for real-world applications of relativistic optics, such as electron/ion acceleration and neutron and positron production. (C) 2002 MAIK "Nauka/Interperiodica".
\end{abstract}

\section{INTRODUCTION}

Over the past 15 years, we have seen a revolution in the generation of ultraintense laser pulses [1]. Presentday lasers can produce pulses with intensities five to six orders of magnitude greater than those previously possible, giving access to new physical regimes. One of these regimes is the so-called "relativistic regime," where the quiver energy of the electrons is equal to or greater than their rest-mass energy [2].

As in the 1960s, when lasers opened up the field of bound-electron nonlinear optics [3] with the demonstration of harmonic generation, stimulated Raman and stimulated Brillouin scattering, optical Kerr effect, etc., the past decade has revealed a new class of phenomena based on the relativistic character of the electron. This relativistic behavior suggests the possibility of extending the field of laser optics from the $\mathrm{eV}$ to the $\mathrm{MeV} / \mathrm{GeV}$ regime. Let us stress in particular the generation of X-ray and $\gamma$-ray photons [4-6], as well as the generation of energetic particle beams of electrons [7], neutrons [8], and ions [9]. A number of theoretically predicted relativistic effects, such as relativistic selffocusing [10], laser wakefield acceleration (LWFA) [11], quasistatic magnetic field generation by relativistic electron beams accelerated inside self-focusing channels [12], harmonic generation [13, 14], and non-

\footnotetext{
${ }^{1}$ This article was submitted by the authors in English.
}

linear Thomson scattering [15], were also demonstrated experimentally, and $\mathrm{MeV}$ ions and $\gamma$-rays were used for the production of nuclear reactions [16]. Until now, all these experiments were performed with lasers delivering intensities in the relativistic regime without full control over the duration, shape, or spot size of the laser pulse.

The aim of this paper is to propose relativistic optics experiments with truly table-top lasers under highly controlled conditions. We will focus on the coherent aspects of light-matter interaction in the relativistic regime. This implies working with pulses consisting of only a few optical cycles. In order to avoid beam breakup by relativistic self-focusing, we must match the laser input numerical aperture (NA) to the channel NA. This condition requires that the beam be focused to a $1-\lambda$ diameter. Only recently have we proved experimentally that the following three basic conditions can be met simultaneously [17]: (i) relativistic intensities, (ii) pulse duration of a few optical cycles, and (iii) focused spot size of $1 \mu \mathrm{m}$. These conditions are satisfied by the kilohertz chirped pulse amplification (CPA) laser developed at the Center for Ultrafast Optical Science (CUOS); this laser has the advantage of being stable, compact, and relatively inexpensive.

Our experimental regime differs from and complements present studies where experiments are performed with relatively long pulses $(0.1-1 \mathrm{ps})$, large spot sizes 
$(10 \mu \mathrm{m})$, and low repetition rates $(<10 \mathrm{~Hz})$. Our experiments will address electron and ion acceleration, relativistic transparency, relativistic self-focusing, coherent relativistic structures (such as solitons and vortices), coherent Thomson scattering by relativistic electrons, and neutron and positron production.

\section{RELATIVISTIC EFFECTS}

First of all, relativistic effects qualitatively modify the electron dynamics in the field of the electromagnetic wave. From the exact solution of the equations of motion of a charged particle in a propagating planar electromagnetic wave [18], it follows that the transverse component of the generalized momentum of an electron is constant,

$$
\mathbf{p}_{\perp}-e \mathbf{A}_{\perp} / c=\text { const },
$$

and that the energy and the longitudinal component of the momentum are related by

$$
\begin{gathered}
\gamma m_{e} c^{2}-p_{\|} c \\
=\left(m_{e}^{2} c^{4}+p_{\perp}^{2} c^{2}+p_{\|}^{2} c^{2}\right)^{1 / 2}-p_{\|} c=\text { const. }
\end{gathered}
$$

It is convenient to express the laser field amplitude in terms of the normalized vector potential $a=e A / m_{e} c^{2}$, where $A$ is the laser-field vector potential. The value of $a$ can be obtained from the expression $I \lambda^{2} / a^{2}=1.37 \times 10^{18} \mathrm{~W} \mu \mathrm{m}^{2} / \mathrm{cm}^{2}$, where $I$ and $\lambda$ are the laser intensity and wavelength, respectively. In the reference frame where the electron was at rest before the interaction with the laser pulse, the electron kinetic energy $K=m_{e} c^{2}(\gamma-1)$ and momentum $\mathbf{p}$ are given by $[18,19]$

$$
\begin{gathered}
K=m_{e} c^{2} a_{\perp}^{2}(x-c t) / 2, \quad \mathbf{p}_{\perp}=m_{e} c \mathbf{a}_{\perp}(x-c t), \\
p_{\|}=m_{e} c a_{\perp}^{2}(x-c t) / 2 .
\end{gathered}
$$

Here, $\mathbf{a}_{\perp}(x-c t)=e \mathbf{A}_{\perp}(x-c t) / m_{e} c^{2}$. We can see that, for $a>\sqrt{2}$, the electron acquires a relativistic energy and the longitudinal component of its momentum is larger than the transverse component.

For a simple plane wave $\left(\mathbf{A}_{\perp}(x-c t)=\right.$ $-(\mathbf{E} c / \omega) \cos (\omega t-k x))$, the force in the direction of the laser pulse propagation can be expressed as

$$
F_{\|}=-\left(e^{2} / 4 \gamma m_{e} \omega^{2}\right) \frac{\partial}{\partial x}\left\{|\mathbf{E}|^{2}[1-(2 \omega t-2 k x)]\right\} .
$$

This expression shows two forces: one is proportional to $\partial|\mathbf{E}|^{2} / \partial x$ (the ponderomotive force) and the other is spatially oscillating with a period of $\lambda / 2$. In the relativistic regime, where $v \sim c$, the magnetic term in the Lorentz force $(e / c) \mathbf{v} \times \mathbf{B}$ becomes as important as the electric part, $e \mathbf{E}$, and the resulting force is directed along the propagation direction. These features are the hallmarks of relativistic nonlinear optics.

The enormous oscillating transverse field of the laser pulse acts on the electrons as a longitudinal, fully rectified field. At $10^{19} \mathrm{~W} / \mathrm{cm}^{2}$, this field corresponds to an equivalent electric field of $60 \mathrm{GV} / \mathrm{cm}$. The electrons in the plasma are subjected to this large Lorentz force and are pushed forward leaving the massive ions behind. The associated charge separation produces an electrostatic field $[11,20]$ up to $E_{\text {break }}=m_{e} \omega_{\mathrm{pe}} c \sqrt{2\left(\gamma_{\mathrm{ph}}-1\right)} / e$, corresponding to the Akhiezer-Polovin wave-break limit [2]. Here, $\omega_{\mathrm{pe}}=$ $\sqrt{4 \pi n e^{2} / m_{e}}$ is the electron plasma frequency and $\gamma_{\mathrm{ph}}=$ $1 / \sqrt{1-v_{\mathrm{ph}}^{2} / c^{2}} \approx \omega / \omega_{\mathrm{pe}}$. The accelerating field propagates at the phase velocity $V_{\mathrm{ph}}$, which is equal to the group velocity $c \sqrt{1-\omega_{\mathrm{pe}}^{2} / \omega^{2}}$ of the driving pulse, whereas the accelerated electrons move at $v \approx c$. The maximum electron energy is determined by the accelerating field over a walk-off distance

$$
l_{\mathrm{acc}} \approx \lambda_{p} / 2\left(\mathrm{v}_{\mathrm{ph}} / c-1\right) \approx \lambda_{p}\left(\omega / \omega_{\mathrm{pe}}\right)^{2},
$$

which is the distance over which electrons and photons move out of phase by $\lambda_{p} / 2=\pi c / \omega_{\mathrm{pe}}$. For $a>1$, the dependence of the wakefield amplitude on the driver laser pulse amplitude should be taken into account (see $[20,21])$. Electron energies as large as $100 \mathrm{MeV}$ have been observed, corresponding to accelerating gradients of $2 \mathrm{GeV} / \mathrm{cm}$ [11].

The distribution of the light intensity across the beam changes the electron mass profile. In addition, the ponderomotive force of the light causes a redistribution of the electron density. These two effects change the index of refraction so that it is maximum on the axis, which leads to self-focusing. As a result, the laser beam shrinks to a single-wavelength spot size and the laser intensity increases accordingly. The threshold power for the pulse self-focusing is given by $P_{c}[\mathrm{GW}]=$ $17.3\left(\omega / \omega_{\text {pe }}\right)^{2}$.

Relativistic self-focusing plays an important role in the production of high-energy electron beams inside the self-focusing channel. Once the electrons are accelerated, they can attract the ions behind them and provide a collimated beam of ions. Proton energies up to $30 \mathrm{MeV}$ have been observed, corresponding to accelerating gradients of $60 \mathrm{GeV} / \mathrm{cm}$ [9], which is the highest acceleration gradient observed to date in the laboratory. The large ponderomotive pressure associated with selffocusing is also at the origin of the so-called "hole boring" [22] in fast ignition [23]. The use of the ions accelerated by the laser pulse in order to ignite the thermonuclear fuel provides a novel approach to the fast ignition of fusion targets [24]. 
Relativistic nonlinearities in a plasma interacting with ultrashort high-intensity laser pulses lead to the formation of long-lived slow-propagating coherent structures such as solitons and magnetic vortices. These structures are part of the complex nonlinear interaction between the laser pulse and the plasma and represent the basic ingredients of the long-time plasma behavior in the wake of the laser pulse.

Solitons appear in the form of stable structures where low-frequency electromagnetic radiation is trapped and, together with magnetic vortices and highenergy particles, represent an important channel of conversion of the electromagnetic pulse energy into plasma energy $[25,26]$. In a homogeneous plasma, their propagation velocity is very small; however, in an inhomogeneous plasma, they are accelerated against the density gradient [27] and their electromagnetic energy can thus be extracted and detected experimentally [28].

Vortices appear in the more general context of the generation of a quasistatic magnetic field in the plasma [29]. Superstrong quasistatic magnetic fields in laser plasma have been studied extensively for many years. They are observed in laser-produced plasmas and can affect the plasma dynamics and the laser self-focusing [12].

To perform the experimental studies of relativistic nonlinear optics in the fundamental limit of singlecycle pulse duration and single-wavelength spot size, we will use the recently developed ultrashort laser technology assembled at CUOS. With this technology, the coherent interaction of ultrashort pulses with near-critical-density plasmas can be studied in the relativisticintensity regime. The duration of a $0.8-\mu \mathrm{m}$ laser pulse will be $5-10$ fs (or $2-4$ optical cycles) and the intensity will be $2 \times 10^{18}-5 \times 10^{19} \mathrm{~W} / \mathrm{cm}^{2}$, which corresponds to $a^{2} \simeq 1-25$. The pulse will be focused to a single-wavelength spot size in order to match it to the relativistic channel size. The target will be made of thin metallic or $\mathrm{C}-\mathrm{H}$ films $20-50 \mathrm{~nm}$ in thickness. The plasma density will be controlled by exciting the thin target prior to the main pulse arrival by means of a properly timed auxiliary pulse. Experiments will be done in real time (at a repetition rate of $0.3-1 \mathrm{kHz}$ ) in order to optimize the signal-to-noise ratio. Concerning relativistic electrons, the measurements of their energy will be carried out with a magnetic spectrometer. In order to reconstruct the ultrarelativistic electron spectrum, a nuclear activation technique can be used.

Coherent interaction of ultrashort pulses with plasmas will show (i) relativistic self-focusing, (ii) electron and ion acceleration, (iii) coherent Thomson scattering, (iv) relativistic transparency, (v) relativistic solitons, (vi) relativistic electron vortices, and (vii) electronpositron pair production.

\section{THE DESIGN OF LABORATORY EXPERIMENTS FOR THE STUDY OF RELATIVISTIC NONLINEAR OPTICS}

\subsection{Relativistic Self-Focusing: Study of the Optimal Coupling of the Laser in the Waveguide Fundamental Mode}

For our laser output equal to $1 \mathrm{~mJ}$ in $10 \mathrm{fs}$, corresponding to a power of $100 \mathrm{GW}$, relativistic self-focusing occurs in plasmas with densities greater than $0.17 n_{\text {cr }}$. In order to avoid the breakup of the beam into filaments, we propose matching the numerical aperture of the input optics to the numerical aperture of the relativistic channel. The plasma index of refraction inside the self-focusing channel is given by $n(r)=$ $\sqrt{1-\omega_{\mathrm{pe}}^{2}(r) / \gamma(r) \omega^{2}}$ with $\gamma(r)=\sqrt{1+a^{2}(r) / 2}$ and $\omega_{\mathrm{pe}}(r)=\sqrt{4 \pi e^{2} n(r) / m_{e}}$. An expansion of the refractive index $n(r) \approx 1-\omega_{\text {pe }}^{2}(r) / 2 \gamma(r) \omega^{2}$ corresponds to a simple waveguide with a quadratically varying index $n(r)=$ $n(0)-\alpha^{2} r^{2} / 2$, where $\alpha=\left(\omega_{\mathrm{pe}} / \gamma\right)$ " near the axis. From waveguide theory, we can show that the optimum numerical aperture for the relativistic waveguide is given by $N A=\omega_{p 0} / \omega$, where $\omega_{p 0}$ is the plasma frequency at low intensities. For $\left(\omega / \omega_{p 0}\right)^{2} \approx 5$, we have $N A \approx 0.4$, which corresponds to a waveguide diameter of $\approx \lambda$ or $0.8 \mu \mathrm{m}$. We can obtain this spot size by using an $f / 1$ parabola corrected with a deformable mirror. For a given laser wavelength, the value of the channel numerical aperture determines the plasma density for the rest of the experiments.

In order to verify that this optimal coupling is indeed obtained for these ultrashort pulses when the numerical apertures are matched, we have performed two-dimensional (2D) particle-in-cell (PIC) simulations with the fully relativistic code described in [30]. In Fig. 1, we show the results of the simulations of the laser plasma matching. An underdense plasma slab with density $n$ equal to $0.5 n_{\mathrm{cr}}$ is located in the region $10 \lambda<x<50 \lambda$. The ion-to-electron mass ratio is 1836 . The incident laser pulse is linearly polarized with the magnetic field vector parallel to the $z$ direction and the electric field in the $(x, y)$ plane ( $p$-polarized pulse). The laser pulse is 20 fs long with the wavelength equal to $\lambda=0.8 \mu \mathrm{m}$. It is focused at the plasma-vacuum interface located at $x=10 \lambda$. The width of the focal spot is about $1 \lambda$. Here, the laser intensity is equal to $I=5 \times 10^{19} \mathrm{~W} / \mathrm{cm}^{2}$; i.e., $a=4.8$. In this case, the optimal conditions for the laser-plasma matching are met. Just after the focus, the laser pulse becomes guided due to relativistic selffocusing, as is seen in Fig. 1. Here, we show the distribution of the electromagnetic energy density in the $(x, y)$ plane at $t=15,30,45$, and 60 (here and below, time is in units of the laser field period $2 \pi / \omega$ and coordinates are in units of $\lambda$ ). At $t=50$, the laser pulse has already lost almost all its energy. We also see the hosing of the laser pulse, which was discussed in $[31,32]$. 


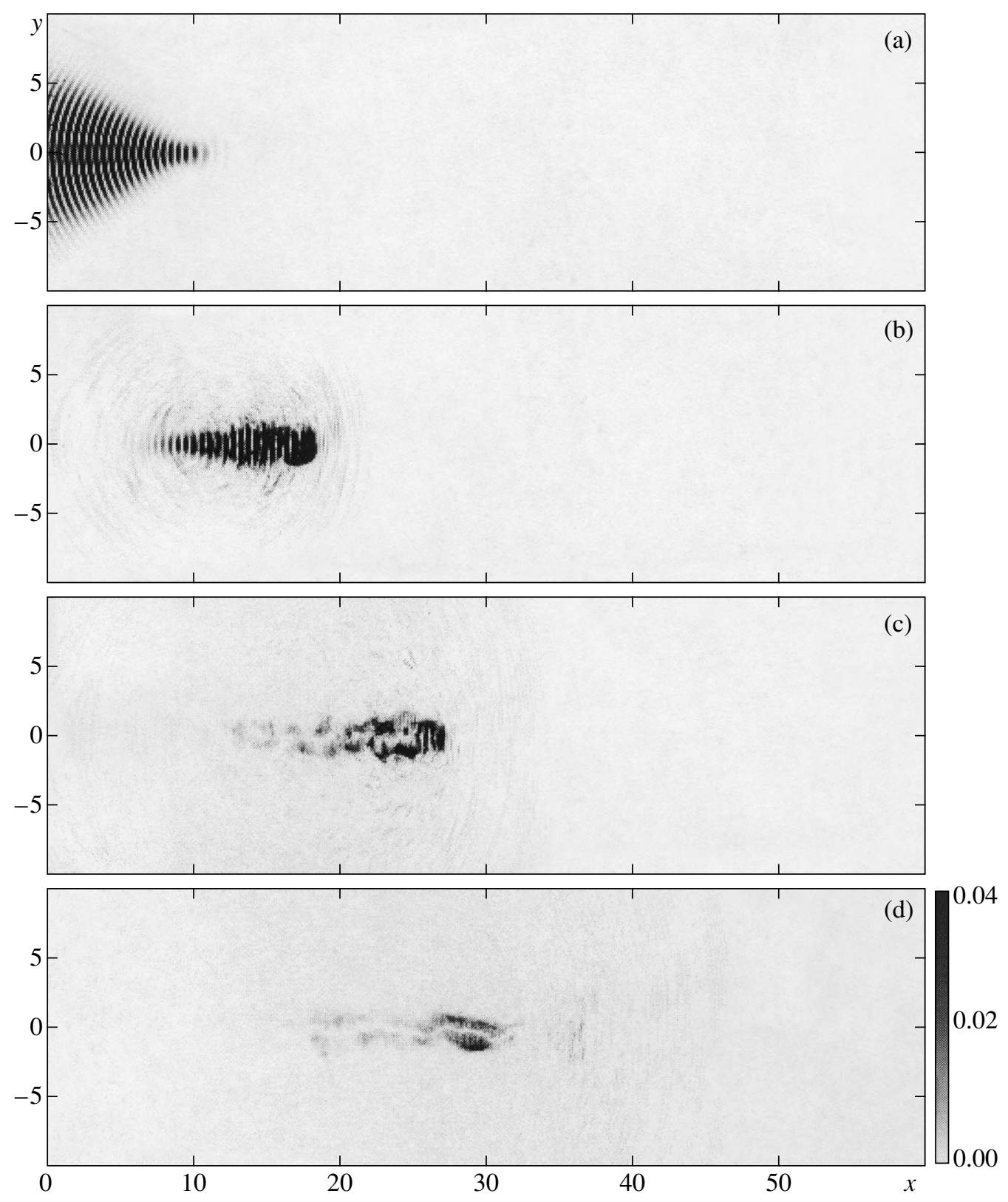

Fig. 1. Distribution of the electromagnetic energy density in the $(x, y)$ plane at $t=$ (a) 15 , (b) 30, (c) 45, and (d) 60 for the case of the optimal matching of a high-intensity $(a=4.8)$ laser pulse with an underdense $\left(n / n_{\mathrm{cr}}=0.5\right)$ plasma.

The ponderomotive pressure of the light forms a channel seen both in the electron and ion density distributions shown in Figs. 2a and 2b. The laser pulse accelerates the electrons predominantly in the forward direction and the ions in the transverse direction. The maximum electron energy is about $12 \mathrm{MeV}$ (Fig. 2c). The ions inside the plasma are accelerated in the transverse direction due to the nonlinear ion wave breaking up to energies of about $0.5 \mathrm{MeV}$ (see Fig. 2d).

A case of nonoptimal laser-plasma matching is obtained by focusing the laser pulse to $5 \lambda$ inside the plasma. Just after the focus, the pulse breaks into sev- eral filaments. As a result, the laser pulse energy depletion is much faster than in the previous case. In addition, instead of a well-pronounced channel, several relatively short and wide channels appear. This nonoptimal regime of laser-plasma matching is shown in Figs. 3 and 4 for the same parameters as in Figs. 1, $2 \mathrm{a}$, and $2 \mathrm{~b}$, except for the focal position. At approximately $t=40$, the pulse has lost most of its energy.

These features, observed in PIC simulations, correlate well with observations of a laser channel produced in a gas jet. This experiment was performed with 400 -fs laser pulses at $\lambda=1 \mu \mathrm{m}$. A laser beam focused to a spot 

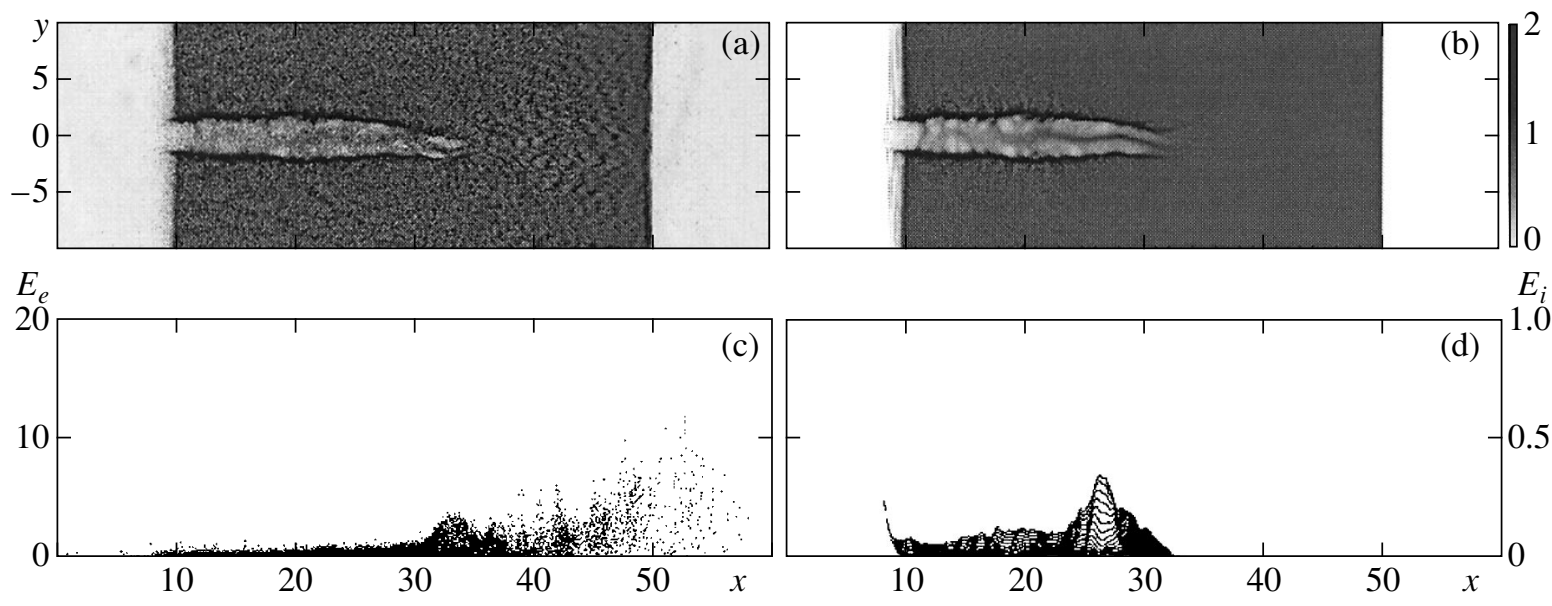

Fig. 2. Distributions of (a) the electron and (b) ion densities in the $(x, y)$ plane and (c) electron and (d) ion kinetic energies inside the channel vs. the $x$ coordinate at $t=70$ for the case of the optimal matching of a high-intensity $(a=4.8)$ laser pulse with an underdense $\left(n / n_{\mathrm{cr}}=0.5\right)$ plasma. The electron and ion densities are in units of $n_{\mathrm{cr}}$, and the electron and ion energies are in units of $m_{e} c^{2}$ and $m_{i} c^{2}$, respectively.

size of about $10 \mu \mathrm{m}$ with an $f / 3.3$ parabolic mirror interacted with a He gas jet with the density $n_{e} \approx 0.08 n_{\mathrm{cr}}$. The experimental setup was described in [33]. Figure 5 shows shadowgrams of a He plasma in the (a) defocusing and (b) relativistic self-focusing and self-channeling dominated regimes for a laser intensity of $6 \times$ $10^{19} \mathrm{~W} / \mathrm{cm}^{2}$ and different distances from the nozzle top. The increase in the distance from the nozzle top corresponds to a less sharp vacuum-gas interface, which leads to a breaking of the laser-plasma matching conditions. The defocusing-dominated regime is characterized by the formation of a short on-axis channel and off-axial laser filamentation. On the other hand, the regime of relativistic self-channeling is characterized by the trapping of a significant portion of the laser beam into a long on-axial plasma channel, which extends to the end of the gas jet.

\subsection{Study of Electron and Ion Acceleration}

3.2.1. Electron acceleration inside the self-focusing channel. Past studies have all dealt with pulses longer than $100 \mathrm{fs}$ and could not differentiate between various mechanisms for electron acceleration. It is expected that, inside the self-focusing channel, the wakefield will not have a regular structure due to the transverse inhomogeneity of the plasma density and the wakefield amplitude, which cause the transverse wave breaking [34]. Nevertheless, fast electrons are generated inside the channel, as has been seen in experiments and computer simulations. For example, PIC simulations performed in [35] seem to indicate a fairly high rate of electron acceleration in the regime when a wakefield with a regular structure is not generated. The electron acceleration under such conditions can be due to a nonlinear interplay of wakefield breaking [36], direct laser acceleration (DLA) [35, 37], betatron-resonance acceleration by the electromagnetic wave in an inhomogeneous quasistatic magnetic field generated in the self-focusing channel [38], and hosing of the laser pulse [39]. The advantage of using an ultrashort laser pulse in conjunction with a thin target was also stressed in [40], where, using PIC simulations, the electron energy scaling $I$ (instead of $\sqrt{I}$ ) was shown for nearcritical-density plasmas. If we respect the NA conditions found above, the light will be optimally coupled in the waveguide fundamental mode. In this channel, because of the shortness of the pulse, we expect that the first electrons will be driven by DLA, as is seen in Fig. 6 , where the phase planes $\left(x, p_{x, e}\right)$ and $\left(x, p_{y, e}\right)$ of the electrons accelerated by the $p$-polarized pulse are presented. We can see electrons accelerated up to the momentum $p_{x, e} \approx 40 m_{e} c$, which corresponds to an energy of about $20 \mathrm{MeV}$.

The CUOS experimental arrangement will give us a unique opportunity to resolve the time structure of the electron beam with femtosecond resolution. We can manipulate the electron beam immediately after the point where it is generated by using the ponderomotive potential of a high-intensity synchronized pulse. A cross correlation between the electron pulse and the laser pulse can be obtained. This cross correlation will give us the time structure of the electron beam directly.

We conclude that, for an intensity of $5 \times 10^{19} \mathrm{~W} / \mathrm{cm}^{2}$, the accelerating field can be on the order of $10 \mathrm{GV} / \mathrm{cm}$ and an electron energy of up to $20 \mathrm{MeV}$ will be obtainable at a high repetition rate $(1 \mathrm{kHz})$.

3.2.2. Ion acceleration during the laser pulse interaction with underdense and overdense plasmas. As was demonstrated in previous works [9], it is 


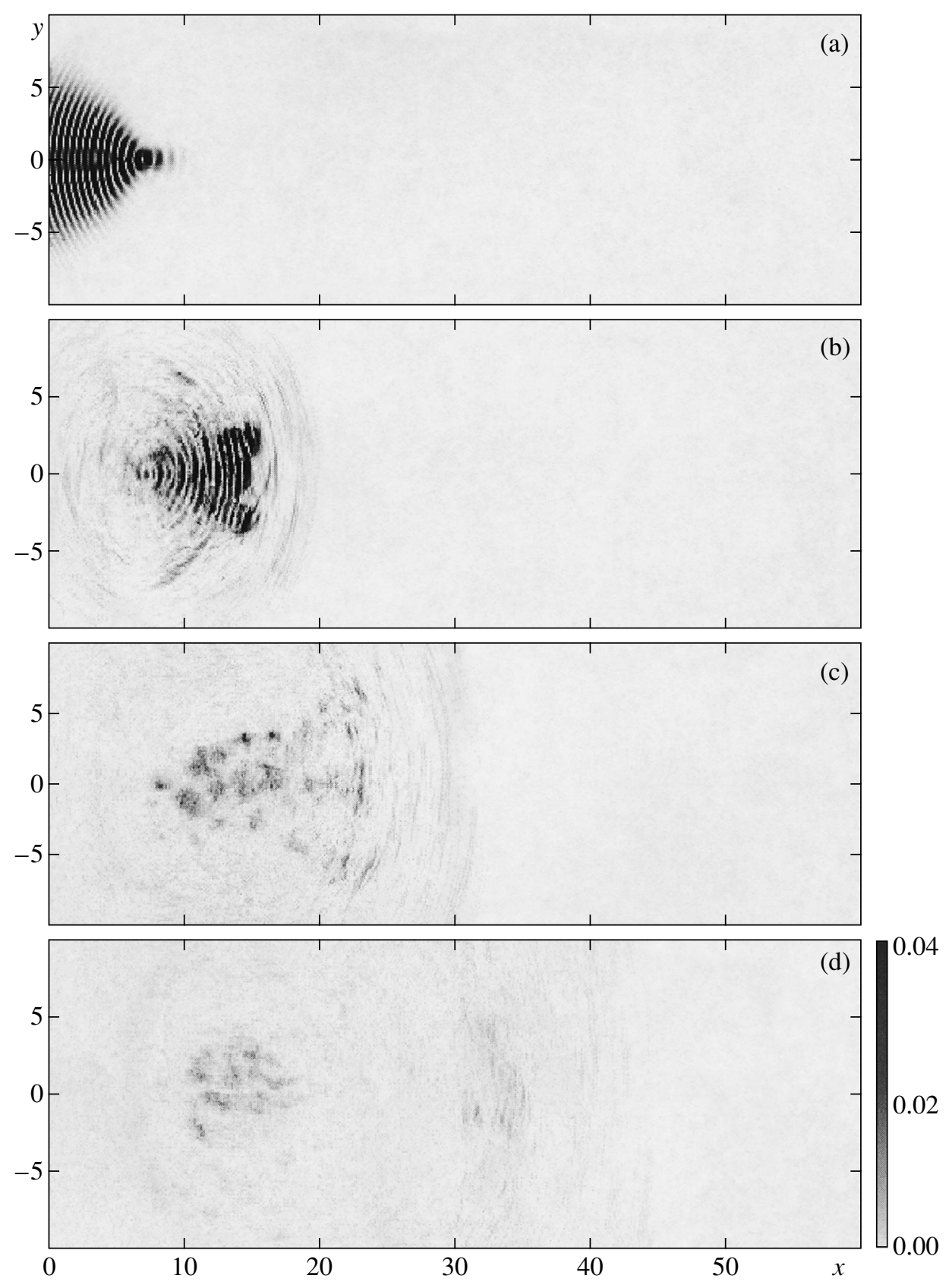

Fig. 3. Same as in Fig. 1 for nonoptimal laser-plasma matching.

expected that the charge separation between electrons and ions will in turn accelerate the ions, thus providing a collimated beam of $\mathrm{MeV}$ ions. The electron beam cone angle $2 \theta$ is typically given by $\tan ^{2} \theta=2 /(\gamma-1)$. Taking advantage of the improved signal-to-noise ratio at kilohertz repetition rates, the time-of-flight of ions will be measured across the electron beam cone. This study will be done as a function of intensity, laser polarization, plasma density, and plasma length.
In Fig. 7, we present the results of the PIC simulations of the interaction of a $20-\mathrm{fs} p$-polarized laser pulse $(a=4.8)$ with a slab of underdense $\left(n / n_{\mathrm{cr}}=0.5\right)$ plasma. We can see that, at $t=200$, the laser pulse has made a channel in the electron (Fig. 7a) and ion (Fig. 7b) densities. The fast ions are localized inside a narrow jet with a length on the order of $10 \mu \mathrm{m}$ and a width of about $1 \mu \mathrm{m}$. The electric field generation and the ion beam collimation appear to be due to the self-generated magnetic field and its interaction with the plasma-vacuum 

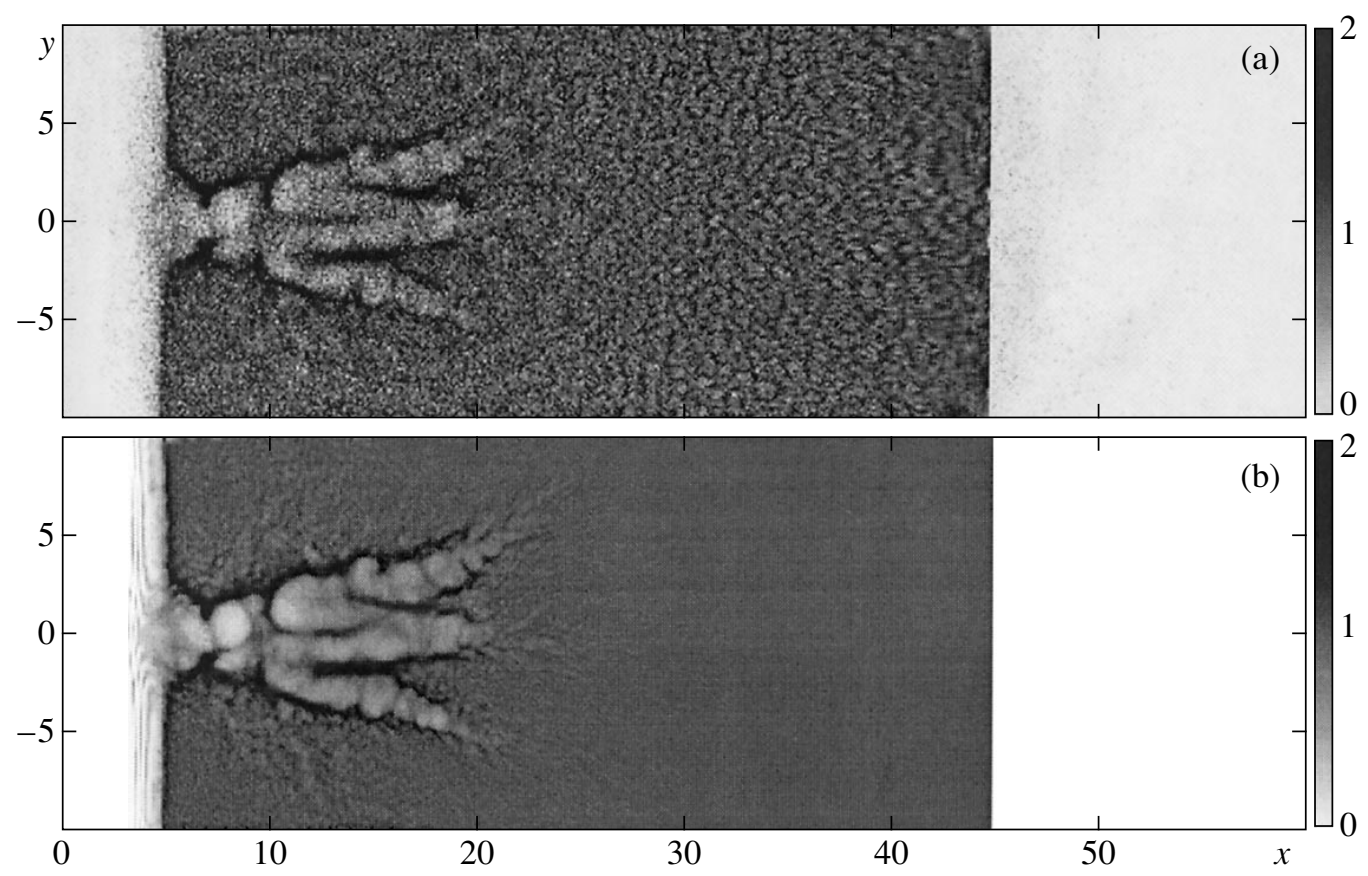

Fig. 4. Same as in Figs. $2 \mathrm{a}$ and $2 \mathrm{~b}$ for nonoptimal laser-plasma matching.

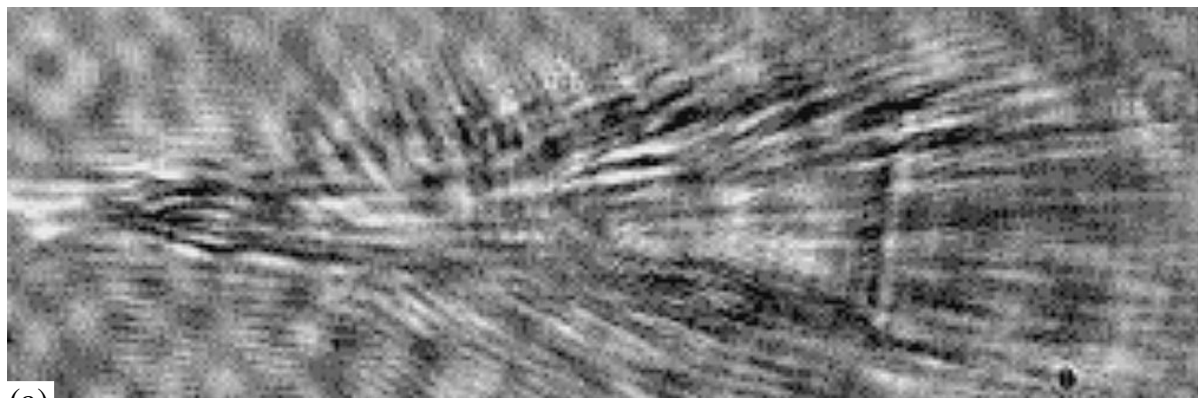

(a)

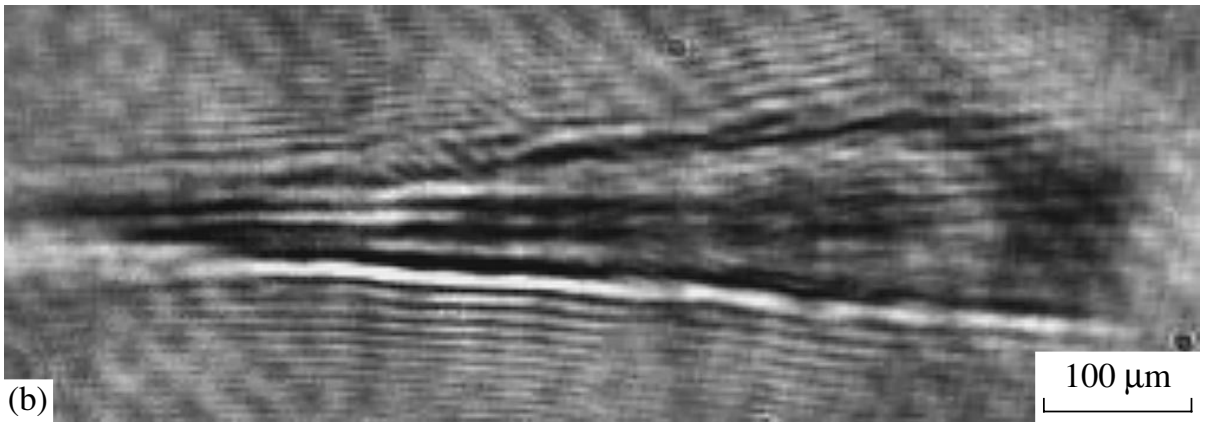

Fig. 5. Shadowgrams of laser interaction with a He jet target for a laser beam focused at a distance of (a) 1.5 and (b) $0.5 \mathrm{~mm}$ from the top of a supersonic gas nozzle. The probe beam is orthogonal to the pump beam and is delayed by $10 \mathrm{ps}$. The high-intensity laser beam propagates from left to right. An external plasma cone is formed due to He ionization by the spatial wings of the laser beam.

interface at the rear side of the plasma slab (see [41] for details). The $x$-component of the ion momentum is equal to $0.1 m_{i} c$, which corresponds to an ion energy of
$5 \mathrm{MeV}$, and the transverse component of the ion momentum is $0.01 m_{i} c$. We find that the emittance of the ion beam is $10^{-4} \mathrm{~mm}$ rad. 


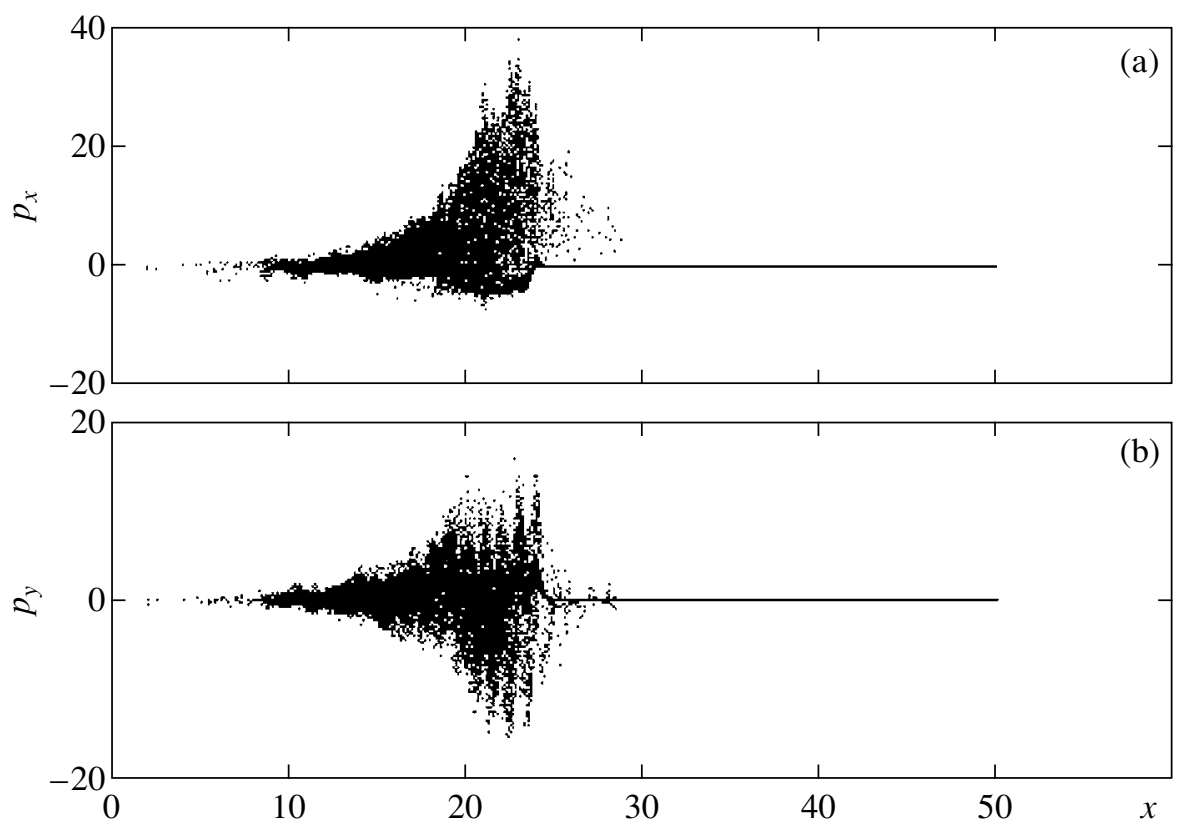

Fig. 6. Phase planes (a) $\left(x, p_{x, e}\right)$ and (b) $\left(x, p_{y, e}\right)$ of the electrons accelerated by a $p$-polarized pulse at $t=40$. The electron momentum is in units of $m_{e} c$.

In order to simulate ion acceleration in an overdense plasma, we studied the interaction of a $p$-polarized laser pulse with an aluminum foil. The foil is assumed to be fully ionized with an ion charge number equal to 13 and atomic weight equal to 27 . The foil thickness is $0.8 \mu \mathrm{m}$, and the foil plasma density is $n=6.5 n_{\text {cr }}$. The foil is localized at $x=10 \lambda$ and is preceded by a $5.2-\mu \mathrm{m}$-long low-density plasma layer, where the density increases exponentially from zero to the critical density on the left-hand side boundary of the foil. A 20-fs-long laser pulse is focused on the foil into a spot with a diameter of $0.8 \mu \mathrm{m}$. At the focal spot, the pulse amplitude is $a=$ 6.8 , which corresponds to the intensity $I=10^{20} \mathrm{~W} / \mathrm{cm}^{2}$. In Fig. 8, we show the distribution of the (a) electron and (b) ion densities in the $(x, y)$ plane and (c) the ion phase plane $\left(x, p_{x, i}\right)$ at $t=200$. We see that the maximum ion energy gain is about $48 \mathrm{MeV}$.

\subsection{Coherent Thomson Scattering}

During the interaction of a relativistically intense electromagnetic wave of amplitude $a \gg 1$ with electrons, the scattered light spectrum contains high harmonics with frequencies up to $\omega a^{3}$ [15]. When the laser pulse is scattered by electron bunches moving with relativistic velocities, a further frequency upshifting can appear. In this way, the scattering of an ultrashort pulse by electrons generated with $\gamma$ up to 10 can be studied (at $I \simeq 5 \times 10^{19} \mathrm{~W} / \mathrm{cm}^{2}$ if we assume that $\gamma$ scales as the intensity $I$ ). In the backscattering mode, an upshift is expected corresponding to $4 \gamma^{2} \omega$ [42]. Thus, radiation
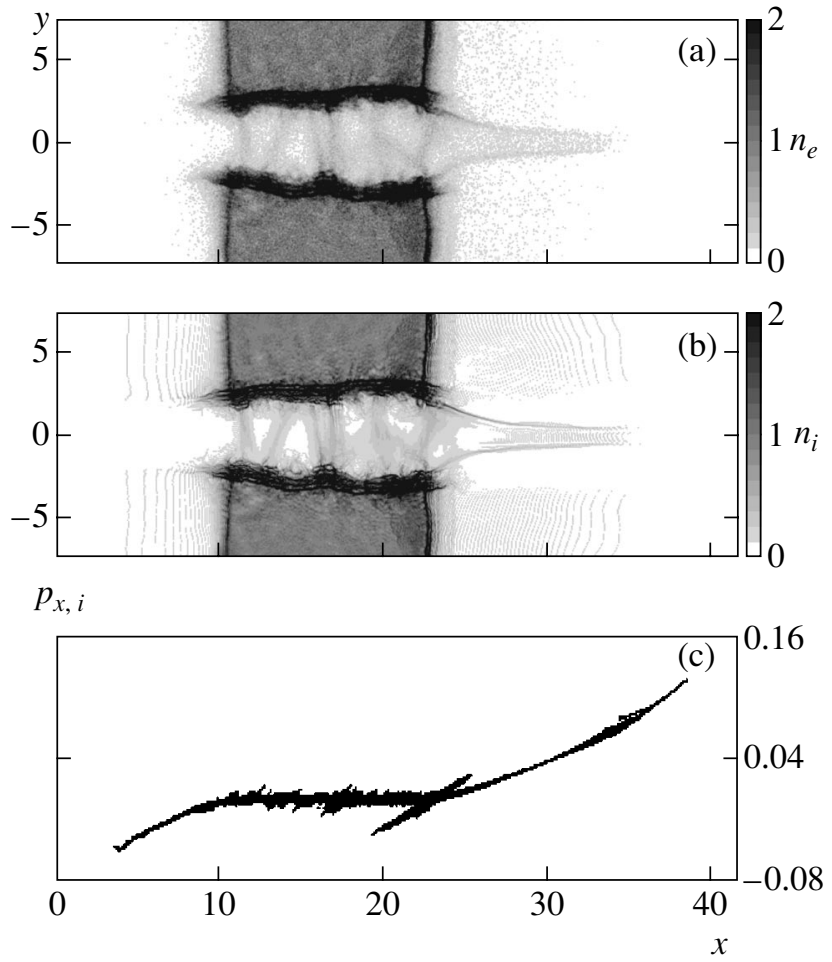

Fig. 7. Interaction of a 20-fs $p$-polarized laser pulse ( $a=$ $4.8)$ with a slab of underdense $\left(n / n_{\mathrm{cr}}=0.5\right)$ plasma. The distributions of the (a) electron and (b) ion densities in the $(x, y)$ plane and (c) the ion phase plane $\left(x, p_{x, i}\right)$ at $t=200$. The electron and ion densities are in units of $n_{\mathrm{cr}}$, and the ion momentum is in units of $m_{i} c$. 

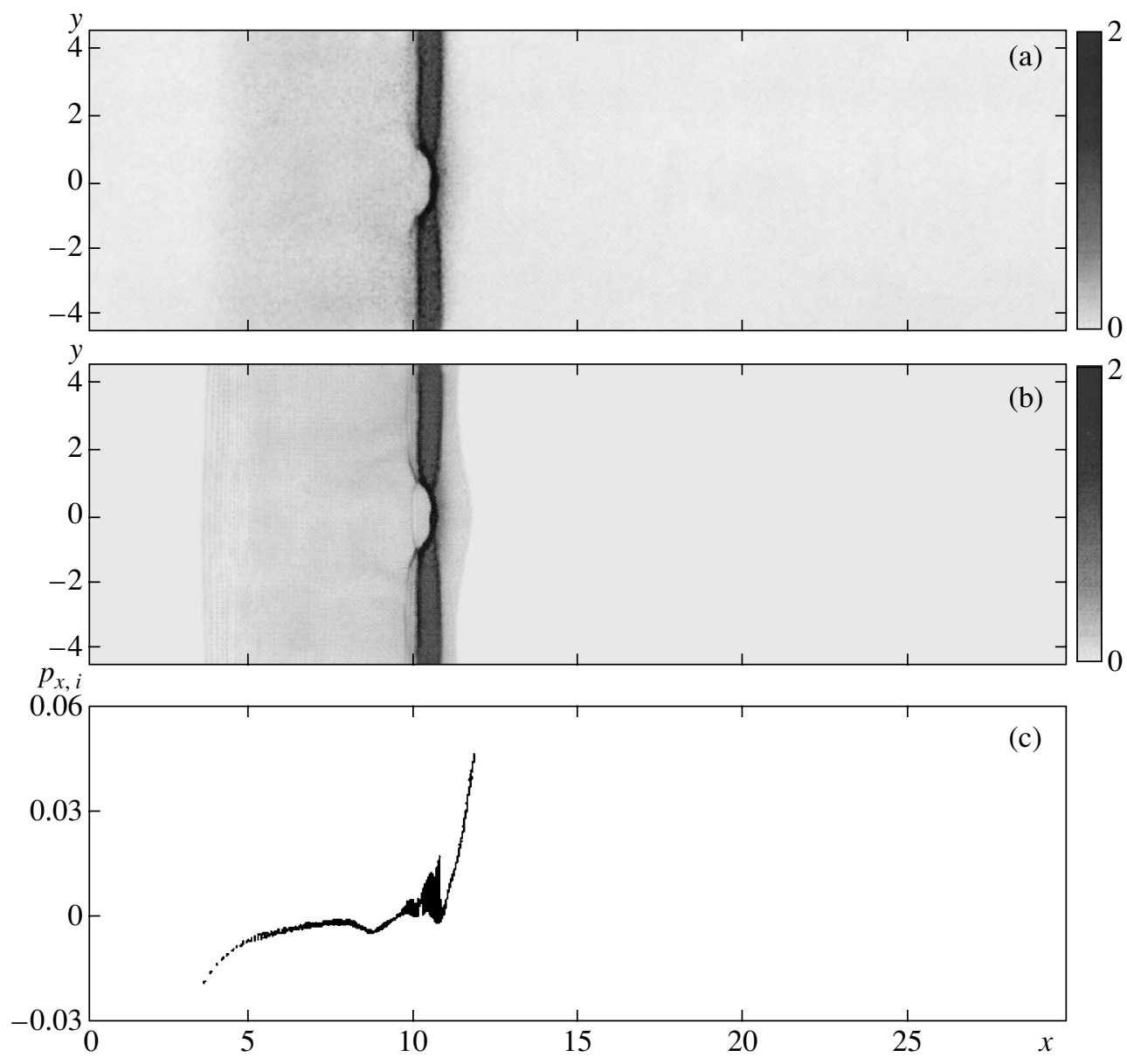

Fig. 8. Interaction of a 20 -fs $p$-polarized laser pulse $(a=6.8)$ with a thin slab of overdense $\left(n / n_{\mathrm{cr}}=6.5\right)$ plasma. The distributions of the (a) electron and (b) ion densities in the $(x, y)$ plane and (c) the ion phase plane $\left(x, p_{x, i}\right)$ at $t=200$. The electron and ion densities are in units of $n_{\mathrm{cr}}$, and the ion momentum is in units of $m_{i} c$.

with a wavelength down to $0.1 \mathrm{~nm}$ can be produced. The intensity of this radiation will be a function of the electron pulse duration. For a duration longer than the laser period (i.e., $3 \mathrm{fs}$ ), the scattered light will scale like $N_{e}$, whereas for pulses shorter than the light period, a coherent scattering is expected with a signal intensity scaling as $N_{e}^{2}$, where $N_{e}$ is the electron density in the electron bunch. This possibility illustrates once more the advantage of using highly controlled pulses limited in time to a single oscillation period and focused over dimensions of a laser wavelength.

We will study the Thomson scattering of a counterpropagating laser pulse by the electron beam. Because of the small transverse size of the electron beam, the electron density will be relatively high (close to $10^{20} \mathrm{~cm}^{-3}$ ). Probing optical pulses will be focused on the electron beam output. The spectrum of the scattered light will be studied as a function of the input light intensity and the density of the accelerated electrons.

\subsection{Relativistic Transparency}

Our high-repetition-rate "relativistic" laser will make possible a detailed study of the propagation of a few-cycle pulses in near-critical-density plasmas. A low-frequency wave can propagate through the plasma if the plasma electrons do not screen the electric field of the wave. The condition for wave propagation requires that the convective electric current $-e n v$ be smaller than the displacement current $\partial_{t} E / 4 \pi$ in the wave; i.e.,

$$
e n_{0} V \leq \frac{\omega E}{4 \pi} .
$$

In the nonrelativistic limit, we have $v \sim e E / m_{e} \omega$ and the transparency condition is equivalent to $\omega>\omega_{\mathrm{pe}}$. In the ultrarelativistic limit $(a \gg 1, v \approx c)$, we can write that the plasma becomes transparent if $\omega>\omega_{\mathrm{pe}} /|a|^{1 / 2}[2$, 43], which corresponds to the lowering of the plasma frequency. Hence, the attenuation distance will scale as $\gamma^{1 / 2}$. This effect significantly changes the transmission 
of a thin film with a thickness comparable to the skin depth. A slab of plasma with a finite length $l$ can screen an electric field not larger than $E=4 \pi n_{e} e l$. We see that, in the case of extremely thin films with thicknesses smaller than the skin depth, the relativistic transparency conditions change [14]. For relativistically strong waves with $a \gg 1$, a film is transparent if $\omega>\omega_{\text {pe }}^{2} l / 2 a c$. This study will require a high-contrast pulse.

We will produce slightly overcritical plasmas. As in the self-focusing study, we will change the plasma density and length by varying the film thickness and the prepulse characteristics, such as delay time and intensity. Then, we will focus on studying relativistic transparency as a function of the plasma parameters (density and length) and the input pulse characteristics (intensity and polarization). The output pulse will be characterized in the spectral and time domains. The timedomain study will be done by cross correlation between the input and output pulses.

\subsection{Relativistic Solitons}

Solitons are a basic ingredient of electromagnetic plasma turbulence, and numerical simulations indicate that they occur in the nonlinear laser plasma interaction [26]. On the basis of the indications provided by the numerical results on soliton formation, we will concentrate on subcycle low-frequency solitons [44]. In a nonuniform plasma, the propagation of the subcycle solitons is strongly affected by the inhomogeneity of the medium. The solitons are accelerated toward the plasma-vacuum interface, where they radiate their energy away in the form of low-frequency electromagnetic bursts during their nonadiabatic interaction with the plasma boundary. These bursts can be used in order to detect subcycle solitons.

Figure 9 presents the results of the 2D PIC simulations of the laser pulse propagation in a homogeneous plasma. The laser pulse is linearly polarized with the electric field vector parallel to the $z$ direction and the magnetic field in the $(x, y)$ plane (s-polarized pulse).
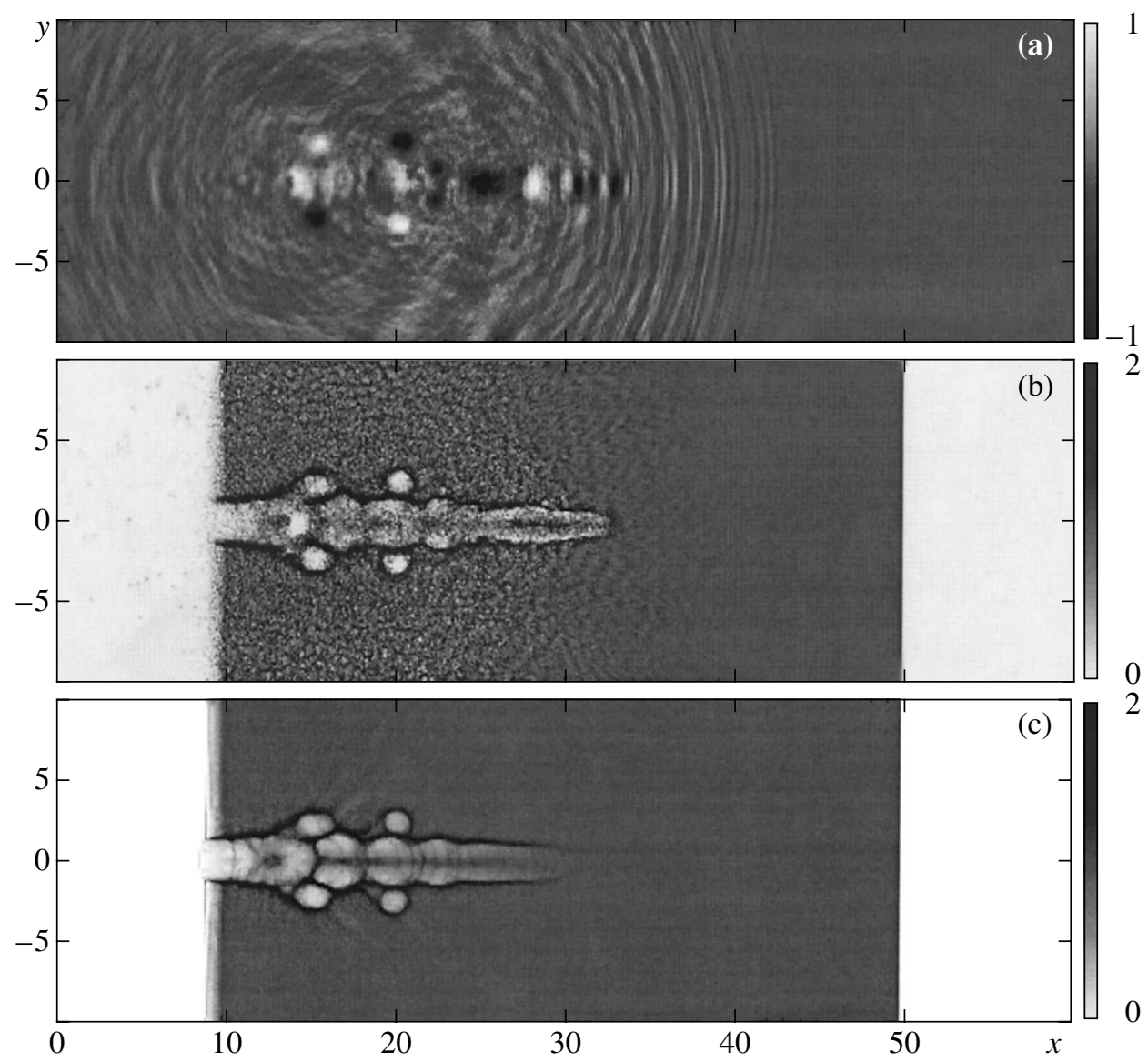

Fig. 9. Generation of solitons by an $s$-polarized pulse. The distributions of (a) the z-component of the electric field and the (b) electron and (c) ion densities in the $(x, y)$ plane at $t=55$. The electric field is in units of $m_{e} c \omega / e$, and the electron and ion densities are in units of $n_{\mathrm{cr}}$. 
The other parameters are the same as in Figs. 1 and 2. Here, we show the distributions of (a) the $z$-component of the electric field and the (b) electron and (c) ion densities in the $(x, y)$ plane at $t=55$. The laser pulse propagates inside the self-focusing channel with a diameter about one-half of the laser wavelength. This makes the pulse amplitude increase up to the value $a_{m} \approx 8$. We can see that the laser pulse leaves behind low-frequency solitons. The solitons are seen as bright spots in the electric field distribution and as bubbles in the electron and ion densities. Rigorously speaking, when the bubbles in the ion density distribution appear, these structures have lost their soliton properties and are called "postsolitons" [45, 46].

We can describe the formation of these postsolitons as follows. Since the soliton formation time is much shorter than the ion response time $t_{i} \approx 2 \pi \sqrt{m_{i} / m_{e}} \omega_{\mathrm{pe}}^{-1}=$ $2 \pi \omega_{\mathrm{pi}}^{-1}$, ions can be assumed to be at rest during the soliton formation. Inside a nonpropagating soliton (subcycle soliton [44]), the maximum electromagnetic field $a_{\max }$ and the soliton frequency $\omega_{s}$ are related as $a_{\max }=2 \sqrt{\omega_{\mathrm{pe}}^{2}-\omega_{s}^{2}} / \omega_{s}$, and the soliton width is equal to $c / \sqrt{\omega_{\mathrm{pe}}^{2}-\omega_{s}^{2}}$. The ponderomotive pressure of the electromagnetic field inside the soliton is balanced by the force due to the charge-separation electric field. The amplitude of the resulting electrostatic potential is given by $\phi=\sqrt{1+a_{\max }^{2}}$. The ponderomotive pressure displaces the electrons outward and the Coulomb repulsion in the electrically nonneutral ion core pushes the ions away. The typical ion kinetic energy corresponds to an electrostatic potential energy on the order of $m_{e} c^{2} a_{\max }$. This process is similar to the so-called "Coulomb explosion" inside self-focusing channels (see [9]). As a result, the bubbles in the ion density distribution are formed.

\subsection{Relativistic Electron Vortices}

In the case of a self-focused laser pulse propagating in an underdense plasma, the fast electron beam is strongly localized in the plane perpendicular to its propagation direction. The separation between the fast electron current and the return current is expected to lead to a strongly inhomogeneous magnetic field. In a 2D model, the magnetic field is essentially dipolar; it consists of two "ribbons" of opposite polarities and vanishes at the axis of the fast electron beam. These two ribbons can be seen as the intersection of the cylindrical magnetic sheet that would be produced in a $3 \mathrm{D}$ configuration by a cylindrical laser pulse with the $z=0$ plane.

A rough estimate of the magnitude of the generated magnetic field can be obtained by observing that the transverse size of the self-focusing channel is on the order of the inverse collisionless electron skin depth $d_{e}=c / \omega_{\mathrm{pe}}$. Since the current density in the channel is given by $j \approx-e n c$, we obtain for the generated magnetic field $e B / m_{e} c \omega \approx \omega_{\mathrm{pe}} / \omega$. For a relativistic laser pulse $(a>1)$ with the wavelength $1 \mu \mathrm{m}$ propagating in a plasma with a near-critical density, the amplitude $B$ of the generated quasistatic magnetic field is extremely large, being on the order of $100 \mathrm{MG}$ or even higher. As a consequence of the equation $\nabla \times \mathbf{B}=-4 \pi e n v / c$, the quasistatic magnetic field in a plasma dominated by the electron dynamics is associated with electron fluid vortices with vorticity $\nabla \times \mathbf{v}=\Delta \mathbf{B} c / 4 \pi e n$. The corresponding electron fluid motion takes the form of an antisymmetric vortex row [29]. The distance between the vortices is comparable to the collisionless skin depth. The vortex row moves as a whole in the direction of the laser pulse propagation with a velocity much smaller than the pulse group velocity.

Inside the vortex, the radial component of the force due to the magnetic pressure and the centrifugal force of the electron rotation is balanced by the force due to the charge-separation electric field [47]. Similar to the case of the postsolitons discussed above, the electrically nonneutral core pushes the ions away and accelerates them. The typical ion energy is also on the order of $m_{e} c^{2} a$.

Two-dimensional PIC simulations of the propagation of linearly polarized laser pulses in a plasma for the same parameters as in Figs. 1-3 show the quasistatic magnetic field generated inside the self-focusing channel. In Fig. 10, we show the distribution of the $z$-component of the magnetic field in the $(x, y)$ plane at $t=70$ for (a) an $s$-polarized pulse and (b) a $p$-polarized pulse.

\subsection{Pair Production}

The kilohertz system is capable of producing intensities in the range of $10^{19} \mathrm{~W} / \mathrm{cm}^{2}$. At these intensities, the ponderomotive potential is higher than $2 m_{e} c^{2}$. This produces conditions for the electron-positron pair generation [48]. Positrons were observed in the interaction of a relatively long and wide high-intensity laser pulse with matter [49]. Therefore, it is possible to produce (in a very controlled way) electron-positron pairs, when a single-cycle laser pulse is focused into a one-wavelength focus spot. The trident pair production has a cross section

$$
\sigma_{T} \cong 9.6 \times 10^{-4}\left(\alpha r_{0} Z\right)^{2}\left(E_{\mathrm{v}} / 2 m_{e} c^{2}-2\right),
$$

where $\alpha$ is the fine structure constant, $r_{0}$ is the classical electron radius, $Z$ is the nuclear charge number, and $E_{v}$ is the electron kinetic energy. For $\gamma=10$ electrons and an interaction volume of $10^{-12} \mathrm{~cm}^{3}$, we can expect to produce as many as $10^{4} \mathrm{pair} / \mathrm{s}$ per one relativistic electron.

This work will depend on our success in producing electrons with $10<\gamma<50$. The efficiency will depend on the $Z$ value of the target material. Positrons will also 

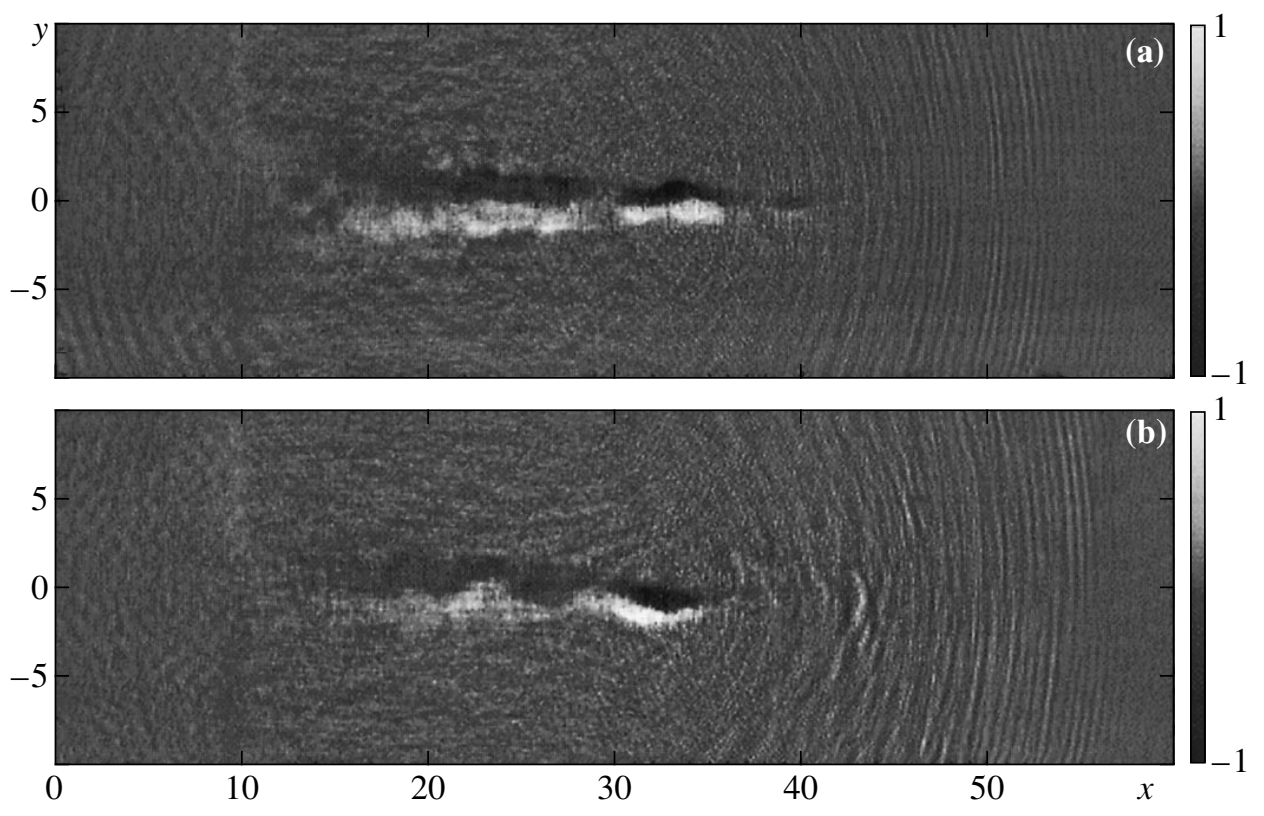

Fig. 10. Distribution of the $z$-component of the quasistatic magnetic field in the $(x, y)$ plane at $t=70$ for the cases of (a) an $s$-polarized pulse and (b) a $p$-polarized pulse. The magnetic field is in units of $m_{e} c \omega / e$.

be analyzed by the time-of-flight technique. Emitted $\gamma$-rays can be detected with our streak camera. Since we know precisely the instant of generation, we can accumulate the signal by using the streak camera in the jitter-free mode to time-resolve the generation processes on the subpicosecond time scale.

\section{EXPERIMENTAL SETUP}

\subsection{The Ultrashort-Pulse Laser}

The experimental study will be carried out using the Ultrafast Photon Source (UPS) Laser Facility at CUOS. The UPS is a high-repetition-rate $0.8-\mu \mathrm{m} \mathrm{Ti}$ : sapphire laser based on the chirped pulse amplification (CPA) design. It is the most powerful sub-10-fs laser and the first kilohertz laser that can produce relativistic intensities.

The following are the features of this laser that make it ideal for the proposed relativistic nonlinear optics studies:

Sub-10-fs pulse. The UPS laser produces 21-fs 3-mJ pulses directly. By using a hollow-core fiber and chirped mirror technique, these pulses are compressed to $8 \mathrm{fs}$ with $\approx 1 \mathrm{~mJ}$ energy. The measured pulse duration is shown in Fig. 11. Work is in progress to obtain 6-fs $1-\mathrm{mJ}$ pulses by optimizing the compression.

Relativistic intensity. The laser can produce focused intensities above $10^{18} \mathrm{~W} / \mathrm{cm}^{2}$ with both 21 -fs and sub-10-fs pulses. The intensity was determined by measuring the focal spot size, pulse duration, and pulse energy. In the case of 21 -fs pulses, an intensity of $>5 \times$
$10^{18} \mathrm{~W} / \mathrm{cm}^{2}$ was confirmed by the observation of charge states up to $\operatorname{Ar}^{13+}$ [50]. We believe that sub-10-fs 1-mJ pulses will be focused to intensities above $10^{19} \mathrm{~W} / \mathrm{cm}^{2}$ in the near future.

Wavelength scale focal spot. In order to generate intensities greater than $10^{18} \mathrm{~W} / \mathrm{cm}^{2}$, an $f / 1$ paraboloid is used to focus the beam. A deformable mirror is used to correct the wave-front distortion from the laser system and to precompensate the aberration caused by the focusing optics. Figure 12 shows the image and line graph of the focal spot. The FWHM spot size is $1.6 \mu \mathrm{m}$, and $78 \%$ of the total energy is inside the $1 / e^{2}$ radius. The actual spot size will be much smaller than that

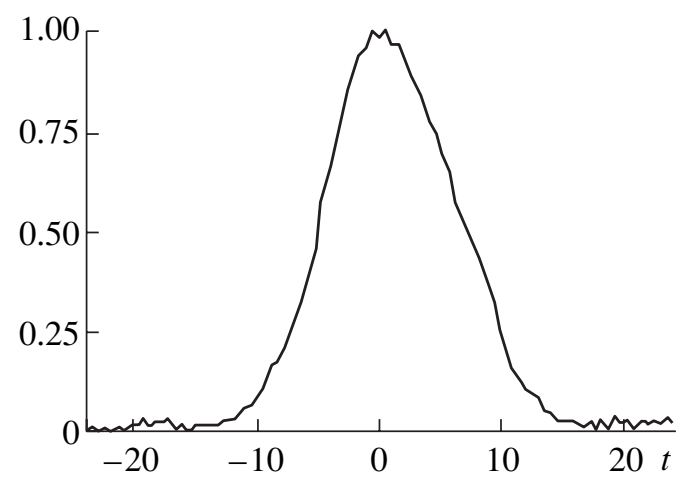

Fig. 11. Autocorrelation trace of the 8 -fs pulse. 

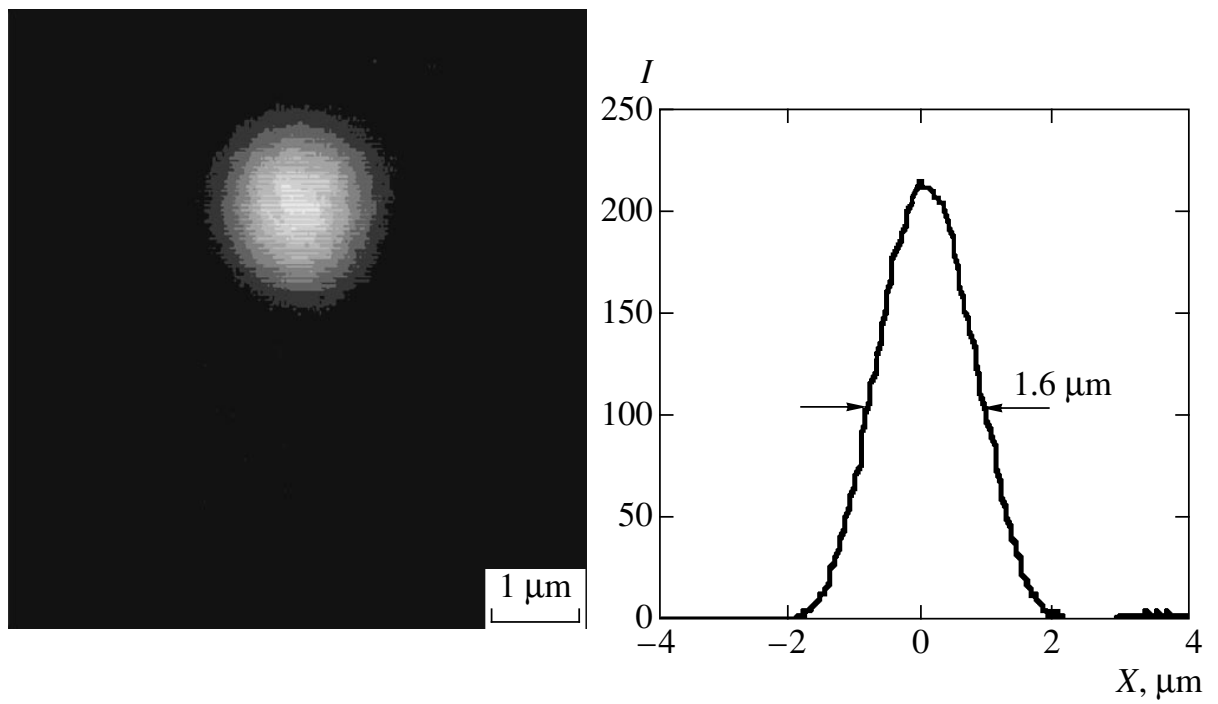

Fig. 12. The image and line graph of a near-diffraction-limited focal spot with a diameter of $1.6 \mu \mathrm{m}$.

shown. The difference is due to aberrations arising in the microscope objective used in our imaging system. The focal spot will match the NA of a relativistic channel, thus favoring the formation of single stable filament.

Marechal's criterion guarantees losses at the focus of less than $20 \%$ provided that the rms wave-front error is less than $1 / 14$. The most efficient way to deliver this wave-front quality is to use a deformable mirror coupled to a wave-front measurement system or a focal intensity feedback loop. While several wave-front measurement systems are available, none offers measurements with a numerical aperture of 0.4 corresponding to $f / 1$ optics. Consequently, we will utilize a genetic algorithm with feedback from the second harmonic generation or from the ionization threshold in gas as a means of optimizing the focal intensity in our laser system.

Using such a system, we have demonstrated the relativistic intensity generation from 21 -fs pulses and the potential to focus 8 -fs pulses to relativistic intensities. In the course of obtaining these results, we have noted that the laser system maintains sufficient stability so that we rarely have to reoptimize the deformable mirror settings.

In addition to the advantages of obtaining a small focal spot and high focal intensity, the deformable mirror also significantly decreases stray light that might cause damage to areas of the target not under investigation.

\subsection{The Target}

In order to delve into the realm of relativistic nonlinearity under the new conditions of high stability, high $\mathrm{NA}$, and a high repetition rate, it will be necessary to use a target that can be replenished within a millisecond. With low-repetition-rate systems, there is no problem in delivering pulses with intensities of $10^{18}$ $10^{20} \mathrm{~W} / \mathrm{cm}^{2}$ to targets of gases, clusters, liquids, or solids. The long time between shots allows plenty of time for target manipulation. At a $1-\mathrm{kHz}$ repetition rate, however, only experiments involving gas and cluster targets have been performed to date. In order to work with near-critical-density plasmas, we propose to manipulate solid thin-film targets to obtain a new specimen for each laser shot. This can only be done without damaging neighboring material because the pulse energy of the $1-\mathrm{kHz}$ laser is in the $1-\mathrm{mJ}$ range.

By using adaptive optics, we will generate a $1-\mu \mathrm{m}$ focal spot with a Gaussian profile. This will be directed toward a target film 10-100 nm in thickness mounted on a support grid. As the experiment continues, the target will be moved to bring new material into place for the next shot. Commercial grids $2 \mathrm{~mm}$ in diameter with square patterns of $12.5-\mu \mathrm{m}$ period are available. The $5-\mu \mathrm{m} \mathrm{Ni}$ grid bars can absorb $150 \mu \mathrm{J}$ of energy without melting and $4.7 \mathrm{~mJ}$ before vaporizing. With the precise focus previously discussed, it will be possible to avoid direct absorption of the laser radiation outside the $7.5 \mu \mathrm{m}$ clear aperture of these cells. Then, with the greatest portion of the laser energy being directed to the target film, the anticipation is that the outermost perimeter of each cell will not melt and resolidification of any melted grid material will occur due to radiation cooling. Thus, proceeding at a $1-\mathrm{kHz}$ rate, the 20000 cells of a target grid will last $20 \mathrm{~s}$. A new grid will be moved into place while background calibrations are carried out. This increase from single-shot to 20000-shot measurements will have the effect of increasing signal-tonoise by a factor of 150 . 
Rapid registration of the target grid will be accomplished by using a video feedback from a full aperture alignment laser transmitted through the target and from a reduced aperture alignment laser monitoring diffraction from the grid. Once a target grid is registered, it will be scanned throughout its aperture.

\section{SUMMARY}

It is only in the last decade that we have been able to produce laser intensities in the relativistic regime. This capability has placed us at the threshold of a fundamentally new regime in nonlinear optics that could be as fruitful as conventional bound-electron nonlinear optics. A host of novel effects have been demonstrated: the generation of X-ray and $\gamma$-ray pulses, the production of high-energy electron and ion beams, the generation of higher harmonics from solids, relativistic self-focusing, nonlinear Thomson scattering, etc. The lasers involved in these studies, although more compact than their predecessors, are still very large and expensive, with energies on the joule level, repetition rates from 0.01 to $10 \mathrm{~Hz}$, and a pulse duration of $\approx 100 \mathrm{fs}$. Owing to progress in short-pulse generation and the application of deformable mirrors for beam focusing, we have recently shown that it is possible to produce relativistic intensities $\left(I>10^{18} \mathrm{~W} / \mathrm{cm}^{2}\right)$ at a $1-\mathrm{kHz}$ repetition rate. The laser pulse energy is in the millijoule range, with sub-ten-femtosecond duration, i.e., in the single-cycle regime. It has a one-wavelength focal spot size. This system is truly table-top and makes the study of lasermatter interaction in a new regime possible. The advantages of operating in this new regime are the following. First, the ultrashort time scale of a few optical cycles will favor a coherent laser-matter interaction. It is expected that electron acceleration in this regime will be proportional to the laser intensity rather than the square root of the intensity. Second, the very small spot size, limited by the laser wavelength, will provide a natural cut-off for spatial instabilities that dominate with larger spot sizes. We, therefore, expect, much like in a single-mode fiber, clean propagation in the plasma and an optimum coupling between the laser beam and the relativistic filament. Third, because of the high repetition rate and enhanced signal-to-noise ratio, we will be able to perform a precise study of laser-matter interaction in this regime. This proposal will concentrate particularly on relativistic self-focusing, relativistic transparency, laser acceleration by optical rectification in plasma (direct laser acceleration), coherent Thomson scattering from accelerated electrons, relativistic solitary waves, relativistic electron vortices associated with the quasistatic magnetic field generation, and positron generation.

To perform this study, we will use the recently developed ultrashort laser technology, assembled at CUOS. Specifically, we will employ compressed millijoule pulses of a few cycles in conjunction with deformable mirrors to reach spot sizes of one micron
$[17,51]$ to develop an accurate understanding of relativistic nonlinear optics. We aim to demonstrate that relativistic nonlinear optics experiments can be carried out with a true table-top laser system, which will provide an inherently superior signal-to-noise ratio.

\section{ACKNOWLEDGMENTS}

This work was supported in part by the USA National Science Foundation through CUOS (contract no. STS PHY8920108) and by the Russian Foundation for Basic Research (project nos. 99-02-16997 and 00-02-16063).

\section{REFERENCES}

1. M. D. Perry and G. Mourou, Science 64, 917 (1994); G. A. Mourou, C. P. J. Barty, and M. D. Perry, Phys. Today 51, 22 (1998); S. Backus, C. G. Durfee III, M. M. Murnane, and H. C. Kapteyn, Rev. Sci. Instrum. 69, 1207 (1998).

2. A. I. Akhiezer and R. V. Polovin, Zh. Éksp. Teor. Fiz. 30, 915 (1956) [Sov. Phys. JETP 3, 696 (1956)].

3. S. A. Akhmanov and R. V. Khokhlov, Nonlinear Optics (VINITI, Moscow, 1965; Gordon and Breach, New York, 1972); N. Bloembergen, Nonlinear Optics: a Lecture Note and Reprint Volume (W. A. Benjamin, New York, 1965; Mir, Moscow, 1966); Y. R. Shen, The Principles of Nonlinear Optics (Wiley, New York, 1984; Nauka, Moscow, 1989); R. W. Boyd, Nonlinear Optics (Academic, San Diego, 1992).

4. J. C. Kieffer, J. P. Matte, H. Pepin, et al., Phys. Rev. Lett. 68, 480 (1992).

5. J. D. Kmetec, C. L. Gordon III, J. J. Macklin, et al., Phys. Rev. Lett. 68, 1527 (1992).

6. F. Beg, A. R. Bell, A. E. Dangor, et al., Phys. Plasmas 4, 447 (1997); P. Norreys, M. Santala, E. Clark, et al., Phys. Plasmas 6, 2150 (1999).

7. C. E. Clayton, K. A. Marsh, A. Dyson, et al., Phys. Rev. Lett. 70, 37 (1993); K. Nakajima, D. Fisher, T. Kawakubo, et al., Phys. Rev. Lett. 74, 4428 (1995); A. Modena, Z. Najimudin, A. E. Dangor, et al., Nature 337, 606 (1995); D. Umstadter, S.-Y. Chen, A. Maksimchuk, et al., Science 273, 472 (1996); R. Wagner, S.-Y. Chen, A. Maksimchuk, and D. Umstadter, Phys. Rev. Lett. 78, 3125 (1997); D. Gordon, K. C. Tzeng, C. E. Clayton, et al., Phys. Rev. Lett. 80, 2133 (1998); S.-Y. Chen, M. Krishnan, A. Maksimchuk, et al., Phys. Plasmas 6, 4739 (1999).

8. G. Pretzler, A. Saemann, A. Pukhov, et al., Phys. Rev. E 58, 1165 (1998); L. Disdier, J. P. Garconnet, G. Malka, and J. L. Miquel, Phys. Rev. Lett. 82, 1454 (1999).

9. G. S. Sarkisov, V. Yu. Bychenkov, V. T. Tikhonchuk, et al., Pis'ma Zh. Éksp. Teor. Fiz. 66, 787 (1997) [JETP Lett. 66, 828 (1997)]; A. G. Zhidkov, A. Sasaki, T. Tajima, et al., Phys. Rev. E 60, 3273 (1999); T. Zh. Esirkepov, Y. Sentoku, K. Mima, et al., Pis'ma Zh. Éksp. Teor. Fiz. 70, 80 (1999) [JETP Lett. 70, 82 (1999)]; S. V. Bulanov, N. M. Naumova, T. Zh. Esirkepov, et al., Pis'ma Zh. Éksp. Teor. Fiz. 71, 593 (2000) [JETP Lett. 71, 407 (2000)]; A. Maksimchuk, S. Gu, K. Flippo, et al., Phys. Rev. Lett. 84, 4108 (2000); 
E. L. Clark, K. Krushelnick, M. Zepf, et al., Phys. Rev. Lett. 85, 1654 (2000); Y. Sentoku, T. V. Lisseikina, T. Zh. Esirkepov, et al., Phys. Rev. E 62, 7271 (2000); S. Wilks, A. B. Langdon, T. E. Cowan, et al., Phys. Plasmas 8, 542 (2001).

10. A. G. Litvak, Zh. Éksp. Teor. Fiz. 57, 629 (1969) [Sov. Phys. JETP 30, 344 (1969)]; C. Max, J. Arons, and A. B. Langdon, Phys. Rev. Lett. 33, 209 (1974); G. Schmidt and W. Horton, Comm. Plasma Phys. Controlled Fusion 9, 85 (1985); P. Sprangle, C. M. Tang, and E. Esarey, IEEE Trans. Plasma Sci. PS-15, 145 (1987); A. B. Borisov, A. V. Borovskiy, O. B. Shiryaev, et al., Phys. Rev. A 45, 5830 (1992); P. Gibbon, P. Monot, T. August, et al., Phys. Plasmas 2, 1305 (1995); S. V. Bulanov, F. Pegoraro, and A. M. Pukhov, Phys. Rev. Lett. 74, 710 (1995); J. Fuchs, Phys. Rev. Lett. 80, 1658 (1998); S.-Y. Chen, G. S. Sarkisov, A. Maksimchuk, et al., Phys. Rev. Lett. 80, 2610 (1998).

11. T. Tajima and J. M. Dawson, Phys. Rev. Lett. 43, 267 (1979); E. Esarey, P. Sprangle, J. Krall, and A. Ting, IEEE Trans. Plasma Sci. 24, 252 (1996).

12. D. W. Forslund, J. M. Kindel, W. B. Mori, et al., Phys. Rev. Lett. 54, 558 (1985); G. A. Askar'yan, S. V. Bulanov, F. Pegoraro, and A. M. Pukhov, Pis'ma Zh. Éksp. Teor. Fiz. 60, 240 (1994) [JETP Lett. 60, 251 (1994)]; A. M. Pukhov and J. Meyer-ter-Vehn, Phys. Rev. Lett. 76, 3975 (1996); M. Borghesi, A. J. Mackinnon, R. Gaillard, et al., Phys. Rev. Lett. 80, 5137 (1998).

13. S. V. Bulanov, N. M. Naumova, and F. Pegoraro, Phys. Plasmas 1, 745 (1994); R. Lichters, J. Meyer-ter-Vehn, and A. M. Pukhov, Phys. Plasmas 3, 3425 (1996); D. von der Linde, AIP Conf. Proc. 426, 221 (1998); M. Zepf, G. D. Tsakiris, G. Pretzler, et al., Phys. Rev. E 58, R5253 (1998).

14. A. V. Vshivkov, N. M. Naumova, F. Pegoraro, and S. V. Bulanov, Phys. Plasmas 5, 2727 (1998).

15. E. S. Sarachik and G. T. Schappert, Phys. Rev. D 1, 2738 (1970); S.-Y. Chen, A. Maksimchuk, and D. Umstadter, Nature 396, 653 (1998); S.-Y. Chen, A. Maksimchuk, E. Esarey, and D. Umstadter, Phys. Rev. Lett. 84, 5528 (2000).

16. K. Nemoto, A. Maksimchuk, S. Banerjee, et al., Appl. Phys. Lett. 78, 595 (2001); K. W. Ledingham, I. Spencer, T. McCanny, et al., Phys. Rev. Lett. 84, 899 (2000); T. E. Cowan, A. W. Hunt, T. W. Phillips, et al., Phys. Rev. Lett. 84, 903 (2000).

17. O. Albert, H. Wang, D. Liu, et al., Opt. Lett. 25, 1125 (2000).

18. L. D. Landau and E. M. Lifshitz, The Classical Theory of Fields (Nauka, Moscow, 1973; Pergamon, Oxford, 1975).

19. H. M. Lai, Phys. Fluids 23, 2373 (1980); B. Rau, T. Tajima, and H. Hojo, Phys. Rev. Lett. 78, 3310 (1997).

20. S. V. Bulanov, V. I. Kirsanov, and A. S. Sakharov, Pis'ma Zh. Éksp. Teor. Fiz. 50, 176 (1989) [JETP Lett. 50, 198 (1989)]; V. I. Berezhiani and I. G. Murusidze, Phys. Lett. A 148, 338 (1990).

21. S. V. Bulanov, F. Califano, G. I. Dudnikova, et al., in Reviews of Plasma Physics, Ed. by V. D. Shafranov (Kluwer, New York, 2001), Vol. 22, p. 227.

22. W. Kruer and S. C. Wilks, Plasma Phys. Controlled Fusion 34, 2061 (1992); S. C. Wilks, W. L. Kruer,
M. Tabak, and A. B. Langdon, Phys. Rev. Lett. 69, 1383 (1992).

23. M. Tabak, J. Hammer, M. E. Glinsky, et al., Phys. Plasmas 1, 1626 (1994).

24. M. Roth, T. E. Cowan, M. H. Key, et al., Phys. Rev. Lett. 86, 436 (2001); H. Ruhl, S. V. Bulanov, T. E. Cowan, et al., Fiz. Plazmy 27, 387 (2001) [Plasma Phys. Rep. 27, 363 (2001)]; V. Yu. Bychenkov, V. Rozmus, A. Maksimchuk, et al., Fiz. Plazmy 27, 1076 (2001) [Plasma Phys. Rep. 27, 1017 (2001)].

25. V. A. Kozlov, A. G. Litvak, and E. V. Suvorov, Zh. Éksp. Teor. Fiz. 76, 148 (1979) [Sov. Phys. JETP 49, 75 (1979)]; P. K. Kaw, A. Sen, and T. Katsouleas, Phys. Rev. Lett. 68, 3172 (1992); D. Farina and S. V. Bulanov, Phys. Rev. Lett. 86, 5289 (2001); Fiz. Plazmy 27, 680 (2001) [Plasma Phys. Rep. 27, 641 (2001)].

26. S. V. Bulanov, I. N. Inovenkov, V. I. Kirsanov, et al., Phys. Fluids B 4, 1935 (1992); S. V. Bulanov, T. Zh. Esirkepov, N. M. Naumova, et al., Phys. Rev. Lett. 82, 3440 (1999).

27. H. Chen and C. Liu, Phys. Rev. Lett. 37, 693 (1976).

28. Y. Sentoku, T. Zh. Esirkepov, K. Mima, et al., Phys. Rev. Lett. 83, 3434 (1999).

29. S. V. Bulanov, M. Lontano, T. Zh. Esirkepov, et al., Phys. Rev. Lett. 76, 3562 (1996); S. V. Bulanov, T. Zh. Esirkepov, M. Lontano, and F. Pegoraro, Fiz. Plazmy 23, 715 (1997) [Plasma Phys. Rep. 23, 660 (1997)].

30. T. Zh. Esirkepov, Comput. Phys. Commun. 135, 144 (2001).

31. G. Shvets and J. S. Wurtele, Phys. Rev. Lett. 73, 3540 (1994); P. Sprangle, J. Krall, and E. Esarey, Phys. Rev. Lett. 73, 3544 (1994); A. G. Litvak, V. A. Mironov, and E. M. Sher, Phys. Rev. E 55, 7441 (1997); B. J. Duda, R. G. Hemker, K. C. Tzeng, and W. B. Mori, Phys. Rev. Lett. 83, 1978 (1999); B. J. Duda and W. B. Mori, Phys. Rev. E 61, 1925 (2000).

32. N. M. Naumova, J. Koga, K. Nakajima, et al., Phys. Plasmas 8, 4149 (2001).

33. G. Sarkisov, V. Yu. Bychenkov, V. N. Novikov, et al., Phys. Rev. E 59, 7042 (1999).

34. S. V. Bulanov, F. Pegoraro, A. M. Pukhov, and A. S. Sakharov, Phys. Rev. Lett. 78, 4205 (1997).

35. C. Gahn, G. D. Tsakiris, A. Pukhov, et al., Phys. Rev. Lett. 83, 4772 (1999).

36. S. V. Bulanov, V. I. Kirsanov, and A. S. Sakharov, Pis'ma Zh. Éksp. Teor. Fiz. 53, 540 (1991) [JETP Lett. 53, 565 (1991)]; M. Raoul, M. Trins, V. Goloviznin, et al., Phys. Rev. E 63, 26406 (2001).

37. J. Meyer-ter-Vehn, Z. M. Sheng, and A. M. Pukhov, Phys. Plasmas 6, 2847 (1999).

38. A. M. Pukhov and J. Meyer-ter-Vehn, Phys. Plasmas 5, 1880 (1998).

39. K.-C. Tseng, W. B. Mori, and T. Katsouleas, Phys. Plasmas 6, 2105 (1999).

40. Wei Yu, V. Bychenkov, Y. Sentoku, et al., Phys. Rev. Lett. 85, 570 (2000).

41. A. V. Kuznetsov, T. Zh. Esirkepov, F. F. Kamenets, and S. V. Bulanov, Fiz. Plazmy 27, 225 (2001) [Plasma Phys. Rep. 27, 211 (2001)]. 
42. P. Sprangle, A. Ting, E. Esarey, and A. Fisher, J. Appl. Phys. 72, 5032 (1992); E. Esarey, S. K. Ride, and P. Sprangle, Phys. Rev. E 48, 3003 (1993).

43. K. Nagashima, Y. Kishimoto, and H. Takuma, Phys. Rev. E 1, 2738 (1970); J. Denavit, Phys. Rev. Lett. 69, 3052 (1992); A. Giulietti, A. Barbini, P. Chessa, et al., Phys. Rev. Lett. 79, 3194 (1997).

44. T. Zh. Esirkepov, F. F. Kamenets, S. V. Bulanov, and N. M. Naumova, Pis'ma Zh. Éksp. Teor. Fiz. 68, 33 (1998) [JETP Lett. 68, 36 (1998)].

45. N. M. Naumova, S. V. Bulanov, T. Zh. Esirkepov, et al., Phys. Rev. Lett. 87, 185004 (2001).

46. H. Ruhl, M. Borghesi, S. V. Bulanov, et al., submitted to Phys. Rev. Lett.
47. A. V. Gordeev and T. V. Loseva, Pis'ma Zh. Éksp. Teor. Fiz. 70, 669 (1999) [JETP Lett. 70, 684 (1999)].

48. J. W. Shearer, J. Garrison, J. Wong, and J. E. Swain, Phys. Rev. A 8, 1582 (1973); E. P. Liang, S. C. Wilks, and M. Tabak, Phys. Rev. Lett. 81, 4887 (1998).

49. C. Gahn, G. D. Tsakiris, G. Pretzler, et al., Appl. Phys. Lett. 77, 2662 (2000).

50. D.-F. Liu, J. Nees, H.-W. Wang, et al., in Proceedings of the Conference on Lasers and Electro-Optics, San Francisco, 2000, Paper CPD-5.

51. J. Queneuille, F. Druon, A. Maksimchuk, et al., Opt. Lett. 25, 508 (2000). 